\title{
A kinetic theory network model to capture first and second order statistics of population activity in large-scale neuronal networks Chin-Yueh Liu* and Duane Q Nykamp
}

\author{
Address: School of Mathematics, University of Minnesota, Minneapolis, MN 55455, USA \\ Email: Chin-Yueh Liu* - liux0518@math.umn.edu \\ * Corresponding author
}

from Eighteenth Annual Computational Neuroscience Meeting: CNS*2009

Berlin, Germany. 18-23 July 2009

Published: 13 July 2009

BMC Neuroscience 2009, I0(Suppl I):PI54 doi:I0.I I86/I47I-2202-I0-SI-PI54

This abstract is available from: http://www.biomedcentral.com/I47I-2202/I0/SI/PI54

(C) 2009 Liu and Nykamp; licensee BioMed Central Ltd.

We present a kinetic theory neuronal network model that captures how first and second order statistics of population activity depend on certain statistical patterns of connectivity. To capture pairwise correlations among neurons in each population, we model the activity of each population by a joint population density $\rho\left(x_{1}, x_{2}, t\right)$, where $\left(x_{1}\right.$, $x_{2}$ ) represents the state of any pair of neurons in the population. We derive the evolution equations of $\rho\left(x_{1}, x_{2}, t\right)$ using the Chapman-Kolmolgorov formalism based on the assumption that the input to any pair of neurons is a multivariate Poisson point process. We then derive the network equations by coupling population equations. The coupling scheme is based on (1) the first and second order statistics of connectivity, (2) the approximation of delayed correlation as instantaneous correlation in each population activity, and (3) the inference of higher order statistics of population activity from lower order statistics via a conditional independence method. We test the validity of our network equations by comparing the numerical solutions to the Monte Carlo simulation under various network configurations. Finally, we discuss the success and failure of the implementation of our kinetic theory network model. 